\title{
Non-Lytic Metastatic Lesion
}

National Cancer Institute

\section{Source}

National Cancer Institute. Non-Lytic Metastatic Lesion. NCI Thesaurus. Code C36038.

A radiologic finding indicating the presence of a metastatic tumor in the bone(s) without associated bone destruction. 